\title{
The Effect of Different Sagittal Angles of the Tibial Guide on Aperture Widening of the Tibial Tunnel during Modified Transtibial Anterior Cruciate Ligament Reconstruction: A Randomized In Vivo Study
}

\author{
Young Chan Kim, $\mathrm{MD}^{1}$, Tulyapruek Tawonsawatruk, $\mathrm{MD}^{2}$, Hyeong Hwa Woon, $\mathrm{MD}^{1}$, Ji Woong Yum, $\mathrm{MD}^{1}$, \\ Myung Jin Shin, $\mathrm{MD}^{1}$, Rodolfo S. Bravo Jr, $\mathrm{MD}^{3}$, and Kyung Wook Nha, MD ${ }^{1}$ \\ ${ }^{1}$ Department of Orthopedic Surgery, Inje University Ilsan Paik Hospital, Goyang, Korea; ${ }^{2}$ Department of Orthopaedic Surgery, Mahidol University, Ramathibodi \\ Hospital, Bangkok, Thailand; 3 Department of Orthopedic Surgery, Far North Luzon General Hospital \& Training Center, Luna, Philippines
}

\begin{abstract}
Purpose: The effect of sagittal plane angle of the tibial tunnel on the severity of tibial intra-articular aperture expansion caused by iatrogenic rereaming in anterior cruciate ligament (ACL) reconstruction using a modified transtibial technique is unknown. The purpose of this study was to compare the severity of intra-articular aperture widening at different angles $\left(40^{\circ}, 45^{\circ}\right.$, and $\left.50^{\circ}\right)$ of the tibial guide (TG).

Materials and Methods: Ninety-seven patients who underwent modified transtibial ACL reconstruction were randomly allocated to TG $40^{\circ}, 45^{\circ}$, and $50^{\circ}$ groups. Intra-articular tibial aperture width (TW) and tibial tunnel length (TTL) were measured intraoperatively using an arthroscopic ruler and a depth gauge.

Results: The TG $50^{\circ}$ group had significantly greater tibial aperture widening than the TG $40^{\circ}$ group. There was a significant difference among TG $40^{\circ}, 45^{\circ}$, and $50^{\circ}$ groups and the percentage of knees with TTL $<35 \mathrm{~mm}$ was $8 \%, 9 \%$ and $3 \%$, respectively. There were 2 females with TTL $<35 \mathrm{~mm}$ in TG $40^{\circ}$ and $45^{\circ}$ groups each. The average mediolateral length of the tibial plateau was $75 \mathrm{~mm}$.

Conclusions: This study shows that the TG angle of $40^{\circ}$ would reduce the severity of intra-articular aperture widening of the tibial tunnel compared to $45^{\circ}$ or $50^{\circ}$ in modified transtibial ACL reconstruction.
\end{abstract}

Keywords: Knee, Anterior cruciate ligament, Reconstruction, Tibial tunnel, Aperture widening

\section{Introduction}

Optimal tunnel placement and graft alignment are important factors associated with successful outcomes of anterior cruciate ligament (ACL) reconstruction ${ }^{1,2}$. However, one of the major

Received June 15, 2016; Revised November 5, 2016;

Accepted December 10, 2016

Correspondence to: Kyung Wook Nha, MD

Department of Orthopedic Surgery, Inje University Ilsan Paik Hospital,

170 Juhwa-ro, Ilsanseo-gu, Goyang 10380, Korea

Tel: +82-31-910-7312, Fax: +82-31-910-7319

E-mail:kwnhamj@hotmail.com

This is an Open Access article distributed under the terms of the Creative Commons Attribution Non-Commercial License (http://creativecommons.org/licenses/by-nc/4.0/) which permits unrestricted non-commercial use, distribution, and reproduction in any medium, provided the original work is properly cited. limitations of the transtibial technique for ACL reconstruction is that the femoral tunnel cannot be placed properly inside the intercondylar notch because of its dependency on the tibial tunnel. Improper alignment and placement of a graft within tunnels created by the transtibial technique may result in graft impingement, graft-tunnel mismatch or tibial aperture widening ${ }^{3-7)}$. The risk of tibial intra-articular aperture expansion caused by iatrogenic re-reaming of the tibial tunnel has been reported as a major disadvantage of the transtibial technique ${ }^{3,8}$, which may also lead to a graft-tunnel mismatch and a delay in incorporation of the graft ${ }^{9}$. In addition, a short tibial tunnel could impair fixation and incorporation of a graft, resulting in a tunnel length-graft length mismatch $^{5)}$.

In the transtibial technique, the direction of the guidewire determined as the optimal trajectory toward the anatomical femoral footprint may cause iatrogenic re-reaming of the tibial tunnel and

www.jksrr.org 
significant intra-articular aperture expansion due to its eccentric and posterolateral positioning within the tibial tunnel ${ }^{3}$.

Several studies have suggested modifications to the coronal plane alignment of the tibial tunnel in the transtibial technique for accurate femoral tunnel positioning and minimal errors ${ }^{4,6,10-12}$. However, the literature is lacking on the effect of variation in the sagittal plane angle of the tibial tunnel in relation to the severity of tibial intra-articular aperture expansion caused by iatrogenic re-reaming in ACL reconstruction using the modified transtibial technique. To the best of our knowledge, there has not been sufficient research suggesting the best sagittal plane angle. Thus, we assumed that the appropriate angle would be between $40^{\circ}$ and $50^{\circ}$ to evaluate the effect of variation in the sagittal plane angle of the tibial tunnel on tibial intra-articular aperture expansion.

The purpose of this study was to investigate the severity of intra-articular aperture widening and proportion of knees with a tibial tunnel length (TTL) less than $35 \mathrm{~mm}$ at different angles of the tibial guide (TG) in ACL reconstruction using the modified transtibial technique. We hypothesized that drilling the tibial tunnel with the TG set at different angles would result in differences in the severity of intra-articular aperture widening of the tibial tunnel.

\section{Materials and Methods}

Initially, 121 patients who underwent primary arthroscopic single bundle ACL reconstruction with a hamstring tendon autograft using the transtibial technique at a single institution were recruited for this prospective, randomized controlled study. This study was approved by the Institutional Review Board of our hospital. The inclusion criteria were: 1) unilateral ACL rupture, 2) age between 18 and 50 years, 3) a normal contralateral knee, and 4) informed consent by patients for participating in this study. The exclusion criteria were concomitant ligament injuries (based on clinical and magnetic resonance imaging examinations) and cartilage degeneration greater than Outerbridge grade 2 (based on intraoperative arthroscopic assessment). Of the 121 patients, 102 patients agreed to take part in the study, 2 of which were excluded from the study due to associated ligament injuries. Ultimately, 100 patients were enrolled in the study and randomly allocated to one of the three groups based on the sagittal angle of $\mathrm{TG}\left(40^{\circ}, 45^{\circ}\right.$, or $50^{\circ}$ group) using a computerized random number table. An envelope was used to conceal the randomization sequence.

Associated meniscal injury was noted at the time of reconstruction in 18 knees in the TG $40^{\circ}$ group, in 19 knees in the TG $45^{\circ}$ group and in 17 knees in the TG $50^{\circ}$ group. Three patients were excluded from the study since cartilage degeneration greater than Outerbridge grade 2 was found on intraoperative arthroscopic assessment. Hence, the final analysis was based on the data obtained from a total of 97 patients: 34 patients (34 knees) in the TG $40^{\circ}$ group, 32 patients ( 32 knees) in the TG $45^{\circ}$ group and 31 patients ( 31 knees) in the TG $50^{\circ}$ group (Fig. 1). There was no statistically significant difference in patient demographics among the three groups (Table 1).

\section{Surgical Technique}

A single surgeon performed the arthroscopic single bundle ACL reconstruction with a hamstring tendon autograft using the transtibial technique in all cases. After diagnostic arthroscopy, any meniscal lesion was treated before reconstruction. Both the semitendinosus and gracilis tendons were harvested using a tendon striper and were prepared as a four-stranded double-looped hamstring autograft. The mean diameter of the double-looped semitendinosus and gracilis tendon autograft was $7.3 \mathrm{~mm}$ (range, 6.5 to $7.9 \mathrm{~mm}$ ). The ACL TG (ConMed Linvatec, Largo, FL, USA) was set at $40^{\circ}, 45^{\circ}$, or $50^{\circ}$ for tibial tunneling according to the group allocation (Fig. 2). The starting point was uniformly created in the coronal plane of the tibial tunnel at midway between the posterior cortex of the proximal tibia and the medial margin of the tibial tuberosity to minimize the effect of coronal orientation of the guide angle on the size of tibial aperture. The intra-

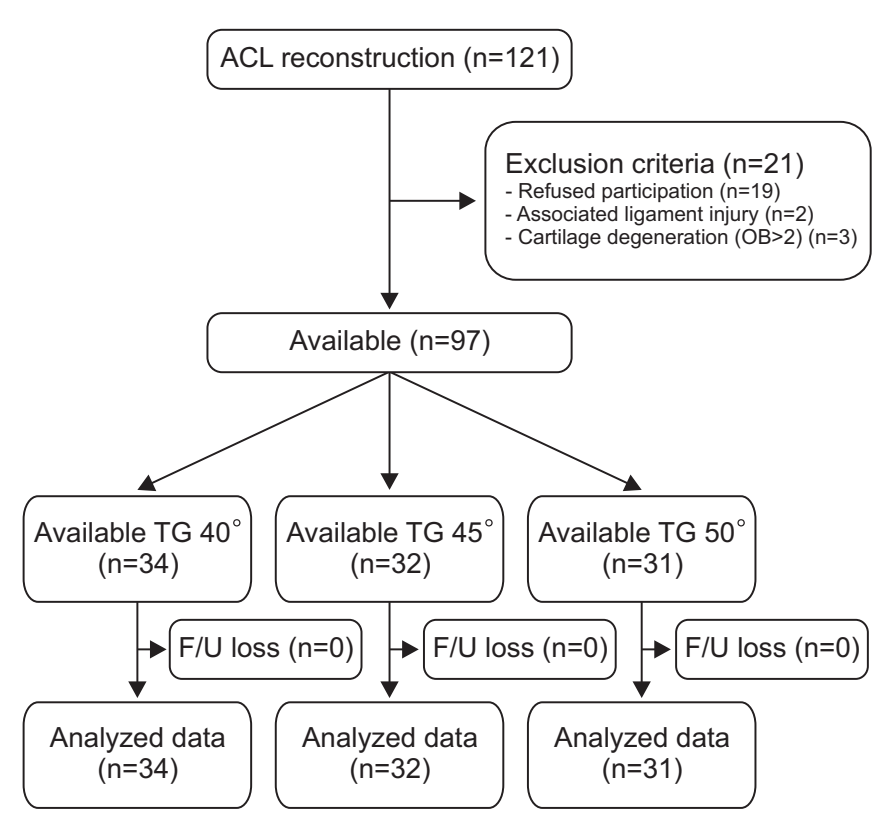

Fig. 1. Flow diagram of the study. ACL: anterior cruciate ligament, OB: Outerbridge, TG: tibial guide, F/U: follow-up. 
Table 1. Comparison of Demographic Characteristics and Mediolateral Width of the Proximal Tibia among the TG $40^{\circ}$, $45^{\circ}$, and $50^{\circ}$ Groups

\begin{tabular}{|c|c|c|c|c|}
\hline Parameter & TG $40^{\circ}$ & TG $45^{\circ}$ & TG $50^{\circ}$ & p-value \\
\hline No. of female subjects & 6 & 6 & 6 & 0.75 \\
\hline Age (yr) & $31 \pm 11(17-30)$ & $38.5 \pm 15(17-75)$ & $36 \pm 11(17-59)$ & 0.06 \\
\hline Height $(\mathrm{cm})$ & $171 \pm 8(152-184)$ & $168 \pm 8(151-182)$ & $171.5 \pm 7.5(150-182)$ & 0.29 \\
\hline Weight (kg) & $71 \pm 13(50.0-99.0)$ & $70.5 \pm 12(50.0-90.0)$ & $73 \pm 8.6(53.8-86)$ & 0.70 \\
\hline Meniscus procedure (meniscectomy/repair/none) & $10 / 6 / 18$ & $14 / 5 / 13$ & $10 / 7 / 14$ & 0.73 \\
\hline Mediolateral size (mm) & $84 \pm 6(71.3-97.0)$ & $84.5 \pm 7(70.0-95.4)$ & $86 \pm 7(68.0-95.5)$ & 0.49 \\
\hline
\end{tabular}

Values are presented as mean \pm standard deviation (range). $\mathrm{p}<0.05$ is statistically significant.

TG: tibial guide.

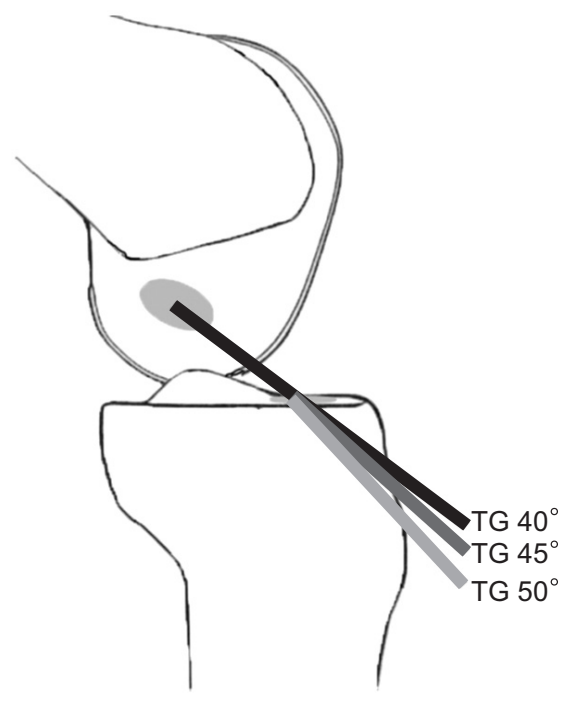

Fig. 2. Schematic drawing showing the sagittal plane angle of the tibial guide (TG) at $40^{\circ}, 45^{\circ}$, and $50^{\circ}$.

articular point of the TG was placed at the center of the native tibial footprint of the ACL. To minimize errors and standardize the orientation of TG, the tip of TG was maintained perpendicular to the tibial slope under fluoroscopic guidance before drilling the guidewire (Fig. 3). The diameter of the tibial and femoral reamers used was the same as that of the graft and a $7 \mathrm{~mm}$ offset size of the femoral guide was used.

After reaming of the tibial tunnel, the longest axis of the intraarticular tibial aperture width (TW) was measured using an arthroscopic ruler (ConMed Linvatec) under direct arthroscopic visualization (Fig. 4). The position of the ruler was aligned parallel to the tunnel aperture and was confirmed under arthroscopic visualization. Similarly, TTL was also measured at the shortest aspect of the tunnel using a depth gauge. In the position of $90^{\circ}$ knee flexion and neutral tibial rotation, the femoral offset aimer (ConMed Linvatec) was then passed through the tibial tunnel, and its hook was placed at around the 10:30 clock position (right

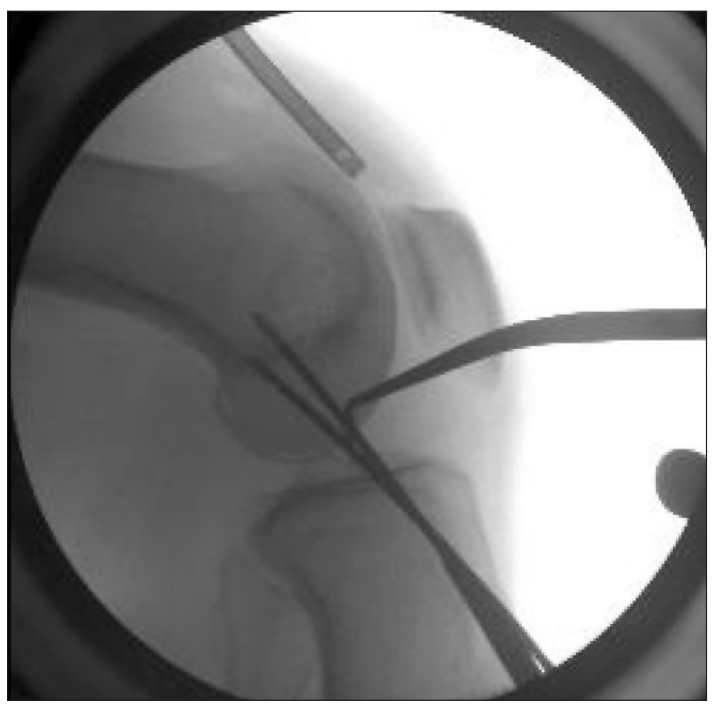

Fig. 3. Intraoperative fluoroscopic image of a tibial guide set at $40^{\circ}$ and $45^{\circ}$. Note that the guide tip is placed perpendicular to the joint surface as confirmed by fluoroscopy.

knee)/1:30 clock position (left knee) at the over-the-top area of the lateral intercondylar notch. This position was maintained for the same femoral tunnel location in all groups. The femoral aiming guide was rotated laterally so that the femoral tunnel could be placed more anatomically ${ }^{12,13)}$. Finally, a $2.4-\mathrm{mm}$ guide pin with a suture eyelet was inserted, and the femoral tunnel was drilled. After reaming of the femoral tunnel transtibially, TW was remeasured using an arthroscopic ruler (Fig. 5). The difference in TW assessed before and after formation of the femoral tunnel was calculated and recorded as tunnel aperture widening. Tibial width was measured as the distance between the medial border and the lateral border of the tibial plateau on the anteroposterior standing $\mathrm{X}$-ray view according to Yoon et al. ${ }^{14)}$. To evaluate the reliability of arthroscopic measurements using the arthroscopic ruler, threedimensional computed tomography (CT) scans (Aquilion One; Toshiba Medical Systems Cor., Otawara, Japan) using PACS view 

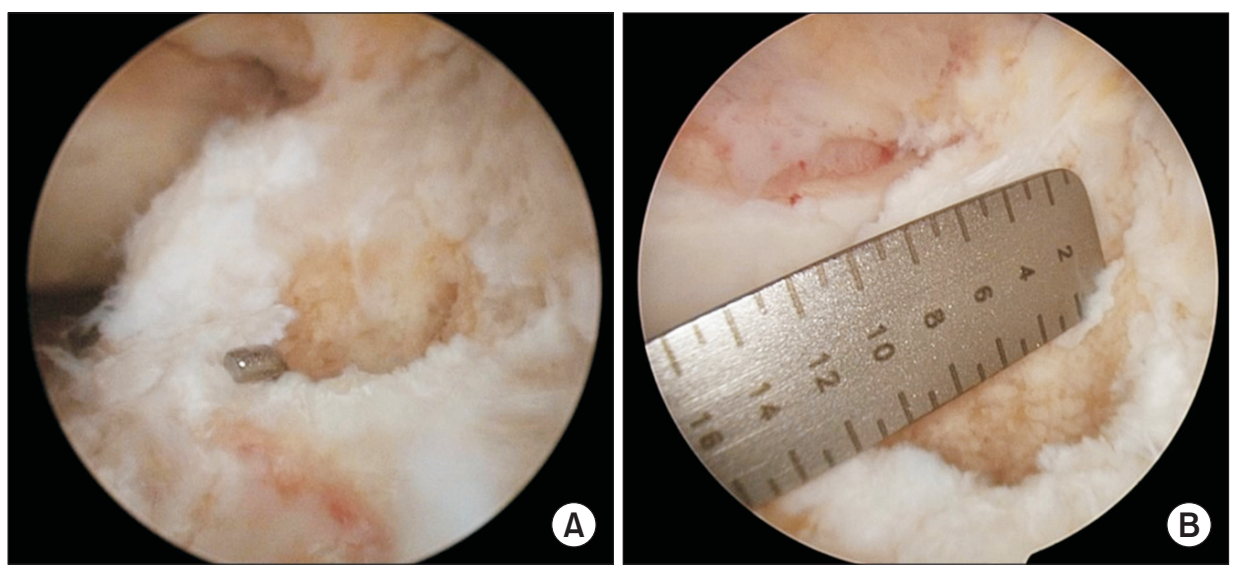

Fig. 4. Measurement of tibial tunnel length (A) and aperture widening (B) during arthroscopy.

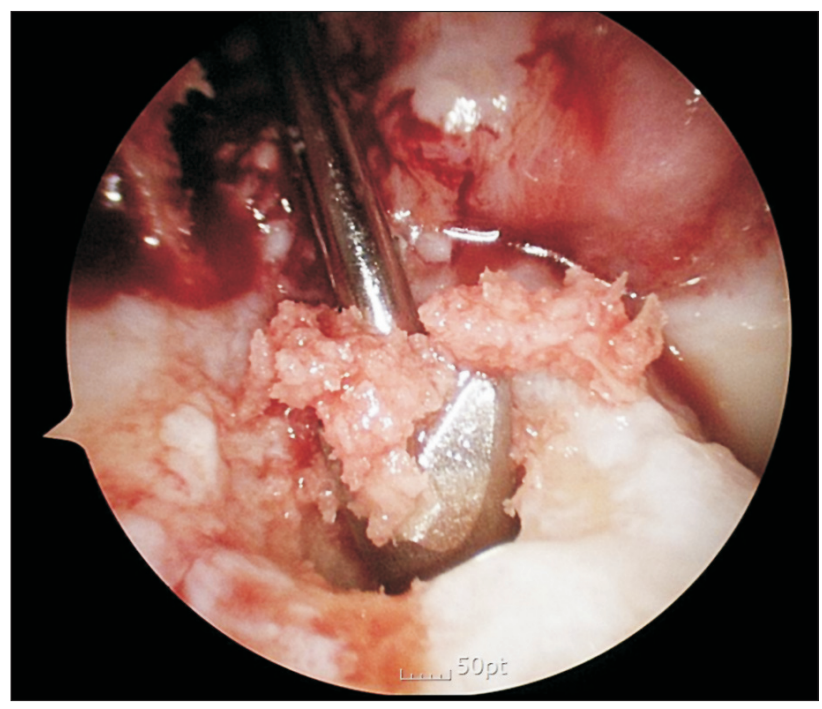

Fig. 5. Arthroscopic image showing eccentric posterolateral re-reaming of the tibial tunnel causing widening of the aperture.

(PACS; Marotech Inc., Seoul, Korea) were evaluated in 20 knees at postoperative 1 week and compared with the arthroscopic measurement values of TW obtained intraoperatively.

The fixation technique for the femoral side and the tibial side was the same in all groups. After passing through the femoral tunnel, the graft was secured using the RigidFix system (DePuy Mitek, Raynham, MA, USA). Once the graft had been secured on the femoral side, the knee was positioned in $10^{\circ}$ flexion and both loops were placed on the Intrafix tie tensioner (DePuy Mitek) with $25 \mathrm{lb}$ force. The tibial end of the graft was then fixed using an Intrafix biodegradable screw (DePuy Mitek) supplemented by a screw and spiked washer.

Patients in all three groups followed an identical accelerated postoperative rehabilitation regimen. Closed chain kinetic exercises were started on the third postoperative day and full weight bearing exercises on the seventh postoperative day. Patients who underwent a concomitant meniscal repair walked on crutches with partial weight bearing for 6 weeks. The goal for all patients was to gain a full range of motion at the end of 6 weeks after surgery. All patients were assessed for function using the International Knee Documentation Committee (IKDC) score and Lysholm score both preoperatively (on admission) and postoperatively (at 2-year follow-up). Knee joint laxity was quantitatively measured using the KT-2000 arthrometer (MEDmetric, San Diego, CA, USA) with a standard manual force of $134 \mathrm{~N}$ applied both preoperatively and postoperatively.

\section{Statistical Analysis}

A power analysis was performed before commencing the study to determine the sample size based on our preliminary data. The sample size was calculated to be 26 subjects in each group for a power of $80 \%$ and a significance level of 0.05 . Wilcoxon rank sum test was used to analyze differences in the average TW between the CT measurement and the arthroscopic ruler measurement. The differences in TW and TTL among the 3 groups of TG $40^{\circ}$, $45^{\circ}$, and $50^{\circ}$ were analyzed using one-way analysis of variance test followed by multi-comparison test using Bonferroni test. The proportions of cases with a TTL less than $35 \mathrm{~mm}$ in the three groups were calculated and then compared by Fisher exact test. Clinical scores (Lysholm score and IKDC score) and KT-2000 measurements (134 N posterior load was applied to the posterior aspect and the anterior translation distance was measured) of patients during preoperative and postoperative evaluation were compared among the 3 groups using Mann-Whitney $U$ test. All statistical analyses were performed with SPSS ver. 18.0 (SPSS Inc., Chicago, IL, USA) and $p$-values of $<0.05$ were considered statistically significant. 
Results

Different TG angles resulted in different TW $(\mathrm{p}=0.01)$ and TTL $(\mathrm{p}=0.03)$. The intra-articular aperture of the tibial tunnel due to iatrogenic re-reaming was approximately 1.5 times greater in the
TG $50^{\circ}$ group (mean, $3.4 \mathrm{~mm}$ ) than in the TG $40^{\circ}$ group (mean, $2.2 \mathrm{~mm})(\mathrm{p}=0.01)$ (Table 2). The aperture width of the TG $50^{\circ}$ group was significantly greater than that of the TG $40^{\circ}$ group in the post hoc test $(p<0.01)$. There were statistically significant differences in TTL among the TG $40^{\circ}$, TG $45^{\circ}$, and TG $50^{\circ}$ groups

Table 2. Comparative Results of Tibial Tunnel Length and Tunnel Aperture Widening

\begin{tabular}{|c|c|c|c|c|}
\hline Parameter & TG $40^{\circ}$ & $\mathrm{TG} 45^{\circ}$ & TG $50^{\circ}$ & p-value \\
\hline Graft size (mm) & $7.1 \pm 0.3$ & $7.2 \pm 0.5$ & $7.3 \pm 0.5$ & 0.43 \\
\hline Tibial tunnel length (mm) & $38.8 \pm 3.1(34-43)$ & $40.1 \pm 2.8(34-45)$ & $42.0 \pm 2.8(34-47)$ & 0.03 \\
\hline Tibial tunnel widening (mm) & $2.24 \pm 1.3(1-8)$ & $2.78 \pm 1.8(1-8)$ & $3.36 \pm 1.4(1-8)$ & 0.01 \\
\hline
\end{tabular}

Values are presented as mean \pm standard deviation (range). $\mathrm{p}<0.05$ is statistically significant.

TG: tibial guide.

Table 3. Comparison of Preoperative and 2-Year Postoperative Clinical Data among the TG $40^{\circ}, 45^{\circ}$, and $50^{\circ}$ Groups

\begin{tabular}{|c|c|c|c|c|c|c|}
\hline \multirow{2}{*}{ Parameter } & \multicolumn{2}{|c|}{ TG $40^{\circ}$} & \multicolumn{2}{|c|}{ TG $45^{\circ}$} & \multicolumn{2}{|c|}{ TG $50^{\circ}$} \\
\hline & Preop & 2-yr F/U & Preop & 2-yr F/U & Preop & 2-yr F/U \\
\hline No. of patients & \multicolumn{2}{|c|}{34} & \multicolumn{2}{|c|}{32} & \multicolumn{2}{|c|}{31} \\
\hline Lysholm score & $61.2 \pm 9.8$ & $93.3 \pm 3.5$ & $58.3 \pm 8.5$ & $90.2 \pm 8.3$ & $59.5 \pm 10.4$ & $92.8 \pm 5.5$ \\
\hline IKDC score & $40.6 \pm 6.3$ & $77.9 \pm 6.5$ & $38.8 \pm 4.7$ & $76.3 \pm 6.1$ & $41.9 \pm 5.7$ & $76.1 \pm 5.6$ \\
\hline KT-2000 (side-to-side difference) & $8.2 \pm 2.6$ & $1.7 \pm 1.5$ & $7.8 \pm 1.8$ & $1.5 \pm 1.2$ & $8.5 \pm 2.2$ & $1.8 \pm 1.4$ \\
\hline
\end{tabular}

Values are presented as mean \pm standard deviation.

TG: tibial guide, Preop: preoperative, F/U: follow-up, IKDC: International Knee Documentation Committee.
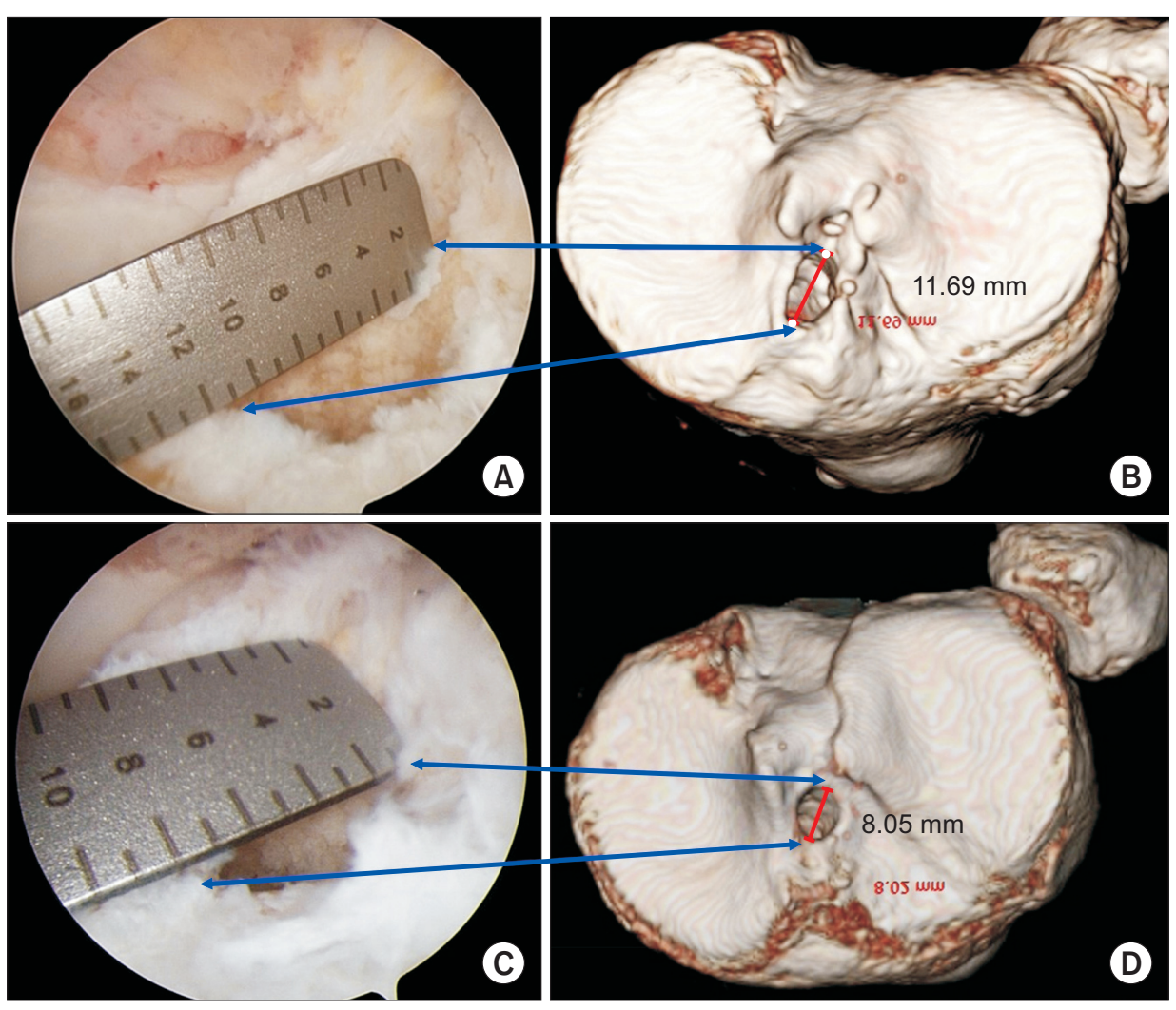

Fig. 6. Measurement of the tibial aperture using an arthroscopic ruler (A, C) and three-dimensional computed tomography (B, D). 
$(\mathrm{p}=0.03)$ (Table 2). The number of knees with TTL measuring less than $35 \mathrm{~mm}$ was $3(8 \%)$ in the TG $40^{\circ}$ group, $3(9 \%)$ in the TG $45^{\circ}$ group and $1(3 \%)$ in the TG $50^{\circ}$ group, showing no statistically significant intergroup difference $(p>0.05)$. There were 2 female patients with TTL measuring less than $35 \mathrm{~mm}$ in the TG $40^{\circ}$ group and TG $45^{\circ}$ group each. The average length of the mediolateral tibial plateau was $75 \mathrm{~mm}$. There were no cases with TTL measuring less than $30 \mathrm{~mm}$ in all groups. Clinical outcomes were not significantly different among the 3 groups (Table 3 ). The average width of the longest axis of the intra-articular aperture measured by the arthroscopic ruler and by CT was $9.52 \mathrm{~mm}$ and $9.48 \mathrm{~mm}$, respectively, showing no statistically significant difference ( $p>0.05)$ (Fig. 6).

\section{Discussion}

The most important finding of this study was that drilling the tibial tunnel with the TG set at $40^{\circ}$ would reduce the severity of intra-articular aperture widening of the tibial tunnel during primary ACL reconstruction using the transtibial technique. The severity of aperture widening was significantly greater after ACL reconstruction performed with the TG set at $50^{\circ}$.

The interface between the tunnel aperture and graft is an important factor in kinematics of a reconstructed ligament ${ }^{8,15)}$. Bedi et al. ${ }^{3)}$ in their cadaveric study reported that iatrogenic posterolateral re-reaming of the tibial tunnel with the TG set at $55^{\circ}$ resulted in expansion of the tibial aperture by $38 \%$ after ACL using the transtibial technique. They attributed such results to the eccentric posterolateral positioning of the femoral guide pin within the tibial tunnel, which was inevitable to optimize the trajectory toward the anatomical footprint of ACL. Our study compared the differences in TW at different sagittal angles of the TG and demonstrated that a greater sagittal angle of the TG caused more widening of the tibial aperture.

Our study also showed that TLL was significantly different among the TG $40^{\circ}, 45^{\circ}$, and $50^{\circ}$ groups. Although the transtibial technique has been reported to produce the most vertical and longest femoral tunnel compared to independent drilling techniques, this technique has been associated with less anatomic positioning of the tunnel ${ }^{7}$. Various techniques designed for better anatomic position of the tunnel may reduce the tunnel length, increasing the risk of postoperative complications such as grafttunnel mismatch and protrusion of the screw inside the joint ${ }^{16)}$. In a cadaveric study, Heming et al. ${ }^{5)}$ attempted to produce tibial and femoral tunnels at the center of the native footprint using the transtibial technique. However, they reported that the technique resulted in shortening of the tibial tunnel (range, 30 to $32 \mathrm{~mm}$ ). In contrast, we adjusted the sagittal angle of the tibial tunnel in this study, which resulted in a relatively long tibial tunnel with a mean length of $38.2 \mathrm{~mm}$ (range, 34 to $37 \mathrm{~mm}$ ). This could be attributable to the difference with the study by Heming et al. ${ }^{5}$ in terms of the coronal angle of the tibial tunnel and slightly posterior target position of the femoral tunnel.

In contrast, a recent study by Wallace et al. ${ }^{17)}$ reported that when the tibial drill guide was set at an angle of $45^{\circ}, 50^{\circ}$, and $55^{\circ}$, the mean TTL was $47.3 \mathrm{~mm}, 48.9 \mathrm{~mm}$, and $50.3 \mathrm{~mm}$, respectively. They concluded that there was no significant difference in TTL in relation to variable tibial drill guide angles because of proximal tibial morphology. They evaluated TTL by measuring the intraosseous length of the guide pin and calculated the mean value in 10 cadaveric specimens. The difference between their results and ours may be due to the difference in the measurement method of the tibial tunnel and the small number of limbs in their study.

This study has some limitations. First, although only the effect of sagittal angle of the TG on aperture widening was evaluated, other factors such as the effect of the starting position of the tibial tunnel were not be evaluated. However, we standardized the starting point to minimize its effect on the outcomes. Second, TW and TTL were measured intraoperatively using a measuring scale or a depth gauge, and inter- and intraobserver variability for measurements was not assessed. Third, although the clinical outcomes were not significantly different among the 3 groups divided based on variation of the sagittal plane angle of the tibial tunnel at the end of 2 year postoperatively, this study did not evaluate any mid- or long-term follow-up differences in clinical outcome among the 3 groups.

\section{Conclusions}

This study shows that the TG set at different angles would affect the severity of intra-articular aperture widening of the tibial tunnel. Setting the TG at $40^{\circ}$ significantly reduced the severity of aperture widening during ACL reconstruction using the transtibial technique.

\section{Conflict of Interest}

No potential conflict of interest relevant to this article was reported. 


\section{References}

1. Jepsen CF, Lundberg-Jensen AK, Faunoe P. Does the position of the femoral tunnel affect the laxity or clinical outcome of the anterior cruciate ligament-reconstructed knee? A clinical, prospective, randomized, double-blind study. Arthroscopy. 2007;23:1326-33.

2. Sommer C, Friederich NF, Muller W. Improperly placed anterior cruciate ligament grafts: correlation between radiological parameters and clinical results. Knee Surg Sports Traumatol Arthrosc. 2000;8:207-13.

3. Bedi A, Musahl V, Steuber V, Kendoff D, Choi D, Allen AA, Pearle AD, Altchek DW. Transtibial versus anteromedial portal reaming in anterior cruciate ligament reconstruction: an anatomic and biomechanical evaluation of surgical technique. Arthroscopy. 2011;27:380-90.

4. Bedi A, Raphael B, Maderazo A, Pavlov H, Williams RJ 3rd. Transtibial versus anteromedial portal drilling for anterior cruciate ligament reconstruction: a cadaveric study of femoral tunnel length and obliquity. Arthroscopy. 2010;26:34250.

5. Heming JF, Rand J, Steiner ME. Anatomical limitations of transtibial drilling in anterior cruciate ligament reconstruction. Am J Sports Med. 2007;35:1708-15.

6. Howell SM, Clark JA. Tibial tunnel placement in anterior cruciate ligament reconstructions and graft impingement. Clin Orthop Relat Res. 1992;(283):187-95.

7. Larson AI, Bullock DP, Pevny T. Comparison of 4 femoral tunnel drilling techniques in anterior cruciate ligament reconstruction. Arthroscopy. 2012;28:972-9.

8. Miller MD, Gerdeman AC, Miller CD, Hart JM, Gaskin CM, Golish SR, Clancy WG Jr. The effects of extra-articular starting point and transtibial femoral drilling on the intraarticular aperture of the tibial tunnel in ACL reconstruction. Am J Sports Med. 2010;38:707-12.

9. Greis PE, Burks RT, Bachus K, Luker MG. The influence of tendon length and fit on the strength of a tendon-bone tunnel complex. A biomechanical and histologic study in the dog. Am J Sports Med. 2001;29:493-7.

10. Howell SM, Gittins ME, Gottlieb JE, Traina SM, Zoellner TM. The relationship between the angle of the tibial tunnel in the coronal plane and loss of flexion and anterior laxity after anterior cruciate ligament reconstruction. Am J Sports Med. 2001;29:567-74.

11. Kopf S, Martin DE, Tashman S, Fu FH. Effect of tibial drill angles on bone tunnel aperture during anterior cruciate ligament reconstruction. J Bone Joint Surg Am. 2010;92:871-81.

12. Rue JP, Ghodadra N, Bach BR Jr. Femoral tunnel placement in single-bundle anterior cruciate ligament reconstruction: a cadaveric study relating transtibial lateralized femoral tunnel position to the anteromedial and posterolateral bundle femoral origins of the anterior cruciate ligament. Am J Sports Med. 2008;36:73-9.

13. Nha KW, Han JH, Kwon JH, Kang KW, Park HJ, Song JG. Anatomical single-bundle anterior cruciate ligament reconstruction using a freehand transtibial technique. Knee Surg Relat Res. 2015;27:117-22.

14. Yoon JR, Kim TS, Lim HC, Lim HT, Yang JH. Is radiographic measurement of bony landmarks reliable for lateral meniscal sizing? Am J Sports Med. 2011;39:582-9.

15. McAdams TR, Biswal S, Stevens KJ, Beaulieu CF, Mandelbaum BR. Tibial aperture bone disruption after retrograde versus antegrade tibial tunnel drilling: a cadaveric study. Knee Surg Sports Traumatol Arthrosc. 2008;16:818-22.

16. Golish SR, Baumfeld JA, Schoderbek RJ, Miller MD. The effect of femoral tunnel starting position on tunnel length in anterior cruciate ligament reconstruction: a cadaveric study. Arthroscopy. 2007;23:1187-92.

17. Wallace M, Bedi A, Lesniak BP, Farrow LD, Ajibade D, Israel HA, Kaar SG. What effect does anterior cruciate ligament tibial guide orientation have on tibial tunnel length? Arthroscopy. 2011;27:803-8. 